\title{
Evaluation of abnormal liver function tests
}

\author{
J K Limdi, G M Hyde
}

Postgrad Med J 2003;79:307-312

Interpretation of abnormalities in liver function tests is a common problem faced by clinicians. This has become more common with the introduction of automated routine laboratory testing. Not all persons with one or more abnormalities in these tests actually have liver disease. The various biochemical tests, their pathophysiology, and an approach to the interpretation of abnormal liver function tests are discussed in this review.

See end of article for authors' affiliations

\section{Correspondence to:}

Dr Jimmy K Limdi

Tameside General

Hospital, Fountain Street,

Ashton-under-Lyne

OL6 9RW, UK

limdi@aol.com

Submitted

10 November 2002

Accepted

12 January 2003

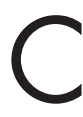
mmonly available tests include alanine transaminase (ALT) and aspartate transaminase (AST), alkaline phosphatase (ALP), gammaglutamyl transferase, serum bilirubin, prothrombin time, or international normalised ratio and serum albumin (box 1 ). They reflect different functions of the liver-that is, to excrete anions (bilirubin), hepatocellular integrity (transaminases), formation and the subsequent free flow of bile (bilirubin and ALP), and protein synthesis (albumin).

Other tests are often performed by a specialist and include hepatitis serology, iron and copper studies, $\alpha_{1}$-antitrypsin levels, and autoantibodies. These relate to the possible aetiology of the abnormality.

The enzymes tested are most commonly raised in liver disease but some enzymes are also present in other tissues and consequently may be raised in other conditions.

When faced with an abnormality in an asymptomatic patient it is imperative to establish that there is an abnormality in the first place, that is statistically significant (the normal value is the mean value in a group of healthy individuals $\pm 2 S D)$. The tests should be repeated and when confirmed appropriate steps be taken.

\section{CLINICAL ASSESSMENT}

A detailed history should be taken and full physical examination performed with a particular emphasis on alcohol consumption, risk factors for viral hepatitis (intravenous drug use, sexual promiscuity, homosexual relations, tattoos, non-

\section{Box 1: Normal values}

- Alanine transaminase: 0-45 IU/I

- Aspartate transaminase: 0-35 IU/I

- Alkaline phosphatase: 30-120 IU/I.

- Gammaglutamyl transferase: 0-30 IU/I.

- Bilirubin: 2-17 $\mu \mathrm{mol} / \mathrm{l}$.

- Prothrombin time: 10.9-12.5 sec.

- Albumin: 40-60 g/l. sterile ear or body piercing, blood or blood product transfusions), medications used currently or previously, herbal or alternative remedies, and occupational exposure to toxins (box 2).

Other factors such as diabetes, obesity and hyperlipidaemia in non-alcoholic fatty liver disease, and family history (for Wilson's disease, haemochromatosis, autoimmune disease) may be significant.

\section{BILIRUBIN}

Bilirubin is formed from the lysis of red cells (the haem component) within the reticuloendothelial system. Unconjugated bilirubin is transported to the liver loosely bound to albumin. It is water insoluble and therefore cannot be excreted in urine. Conjugated bilirubin is water soluble and appears in urine.

Within the liver it is conjugated to bilirubin glucoronide and subsequently secreted into bile and the gut respectively. Intestinal flora breaks it down into urobilinogen, some of which is reabsorbed and either excreted via the kidney into urine or excreted by the liver into the gastrointestinal tract. The remainder is excreted in the stool as stercobilinogen giving stool its brown colour.

Serum bilirubin is normally mainly in an unconjugated form reflecting a balance between production and hepatobiliary excretion. Bilirubin production increases in haemolysis, ineffective erythropoiesis, resorption of a haematoma, and rarely in muscle injury. In all these cases the bilirubin is mainly in an unconjugated form. Conjugated hyperbilirubinaemia characteristically occurs in parenchymal liver disease and biliary obstruction.

\section{UNCONJUGATED HYPERBILIRUBINAEMIA}

Unconjugated hyperbilirubinaemia (indirect bilirubin fraction $>85 \%$ of total bilirubin) occurs with increased bilirubin production or in defects in hepatic uptake or conjugation, which in turn may be inherited or acquired (box 3 ).

Gilbert's syndrome deserves particular mention. This is a common, benign disorder characterised by unconjugated hyperbilirubinaemia, which is exacerbated by fasting. It does not require any specific treatment and the patient should be reassured.

Abbreviations: ALP, alkaline phosphatase; ALT, alanine transaminase; AST, aspartate transaminase; ELISA, enzyne linked immunosorbent assay; $\mathrm{HbsAg}$, hepatitis $\mathrm{B}$ surface antigen; HFE, haemochromatosis gene; $\mathrm{NASH}$, non-alcoholic steatohepatitis 


\section{Box 2: Clinical assessment}

1. Alcohol consumption.

2. Risk factors for viral hepatitis:

- Intravenous drug use.

- Sexual promiscuity.

- Homosexual relations.

- Tattoos.

- Non-sterile ear or body piercing

- Blood or blood product transfusions.

- Residence in developing nations.

3. Medications.

4. Occupational exposure to toxins.

\section{Box 3: Causes of isolated hyperbilirubinaemia}

(A) Unconjugated

1. Increased bilirubin production.

- Haemolysis.

- Ineffective erythropoiesis.

- Blood transfusion.

- Resorption of haematomas.

2. Decreased hepatic uptake.

- Gilbert's syndrome.

- Drugs-for example, rifampicin.

3. Decreased conjugation.

- Gilbert's syndrome.

- Criggler-Najiar syndrome.

- Physiological jaundice of the newborn.

(B) Conjugated

1. Dubin-Johnson syndrome.

2. Rotor's syndrome.

Disproportionate isolated unconjugated hyperbilirubinaemia may also be seen in fulminant Wilson's disease. It is interesting that the ALP is low in such situations. Haemolysis is believed to result from copper release in the blood stream with resulting red cell lysis.

It is worth mentioning here that bilirubin levels of more than $85 \mu \mathrm{mol} / \mathrm{l}$ in the presence of normal hepatic function cannot be explained by chronic haemolysis alone. ${ }^{12}$

\section{CONJUGATED HYPERBILIRUBINAEMIA (DIRECT BILIRUBIN $>50 \%$ OF TOTAL BILIRUBIN)}

This occurs in inherited or acquired defects in hepatic excretion. Bilirubin levels have prognostic significance in alcoholic hepatitis, primary biliary cirrhosis, and in acute liver failure. However a disproportionate rise in conjugated bilirubin has limited diagnostic value. As conjugated bilirubin is excreted in urine, bilirubin levels rarely exceed $510 \mu \mathrm{mol} / \mathrm{l}$ in the absence of renal failure or haemolysis. ${ }^{3}$

\section{AMINOTRANSFERASES}

These include AST and ALT. They are an excellent marker of hepatocellular injury. They participate in gluconeogenesis by catalysing the transfer of amino groups from aspartic acid or alanine to ketoglutaric acid to produce oxaloacetic acid and pyruvic acid respectively.

AST is present in cytosolic and mitochondrial isoenzymes and is found in the liver, cardiac muscle skeletal muscle, kidneys, brain, pancreas, lungs, leucocytes, and red cells. ${ }^{4}$ It is less sensitive and specific for the liver.

ALT, a cytosolic enzyme is found in its highest concentrations in the liver and is more specific to the liver. ${ }^{4}$

Hepatocellular injury and not necessarily cell death is the trigger for release of these enzymes into the circulation.

When faced with an abnormality, the first step should be to assess the degree of abnormality. The tests should probably be repeated if the abnormality is mild. Further investigation is

\section{Box 4: Common causes of raised transaminases}

- Alcohol.

- Medications: non-steroidal anti-inflammatory drugs, antibiotics, HMG Co-A-reductase inhibitors, antiepileptic drugs, antituberculous drugs, herbal medications, illicit drug use.

- Non-alcoholic steatohepatosis.

- Chronic hepatitis B and C.

- Autoimmune diseases.

- Haemochromatosis.

- Wilson's disease.

- Congestive cardiac failure and ischaemic hepatitis.

- $\alpha_{1}$-Antitrypsin deficiency.

- Coeliac disease.

- Endocrine disease: hypothyroidism, Addison's disease.

- Diseases of striate muscle.

- Glycogen storage diseases.

warranted if repeated tests confirm abnormality. Very high levels should prompt further evaluation without delay.

Common causes are non-alcoholic fatty liver disease, alcoholic liver disease, chronic hepatitis B and C, autoimmune liver disease, haemochromatosis, Wilson's disease, $\alpha_{1}-$ antitrypsin deficiency, and coeliac disease.

\section{CAUSES OF RAISED AMINOTRANSFERASES Alcohol}

A reliable history is helpful; in reality this can be difficult. A biochemical clue is the ratio of AST to ALT (2:1 at least), reflecting the low level of activity of ALT in people with alcoholic liver disease.

A gammaglutamyl transferase level of twice the normal with an AST/ALT ratio of 2:1 or more is highly suggestive of alcohol abuse. ${ }^{5}$ Gammaglutamyl transferase is not specific to alcohol and hence cannot be used as an isolated test. ${ }^{4}$

Notably, AST levels of more than eight times normal and ALT levels more than fives times normal are exceptionally rare in alcoholic liver disease. ${ }^{5}$ Conversely ALT may be normal even in the face of severe alcoholic liver disease. Typically aminotransferase levels are less than $300 \mathrm{U} / \mathrm{l}$ in alcohol induced liver injury unless of course other insults such as viral or Paracetamol induced liver injury are superimposed on alcoholic liver disease, when serum aminotransferase levels may exceed $1000 \mathrm{U} / \mathrm{l}$. Very high aminotransferase levels are characteristically seen in ischaemic liver injury, acute viral hepatitis, and drug or toxin induced liver injury and occasionally in acute obstructing choledocholithiasis.

\section{Medication}

Several drugs may cause raised liver enzymes, commonly non-steroidal anti-inflammatory drugs, antibiotics, statins, antiepileptics, and antituberculosis drugs (box 4). A specific inquiry should be made about herbal remedies, alternative medications, and substance abuse. The commonest drug induced cause of a massive aminotransferase rise is paracetamol overdose, typically at doses of $10 \mathrm{~g}$ or more but also at lower doses particularly in the context of other insults such as alcohol.

Drug induced liver enzyme rise may present as an enzyme rise soon after starting a drug. A logical step is to stop the drug and see if the results normalise. If the drug is essential for the patient a risk benefit assessment will be required. A liver biopsy may be necessary to assess the severity of the injury or confirm a drug induced reaction.

\section{Viral hepatitis}

Hepatitis B and/or C are common causes and hepatitis serology should thus be requested. We will consider the diagnostic laboratory features in turn. 
If hepatitis B surface antigen (HbsAg) is positive then HBeAg and HBe antibody results are normally available automatically. A positive HbsAg may represent an acute or chronic infection. HbsAg becomes positive $2-8$ weeks before biochemical evidence of jaundice or liver damage. Once HbsAg has appeared other markers of viral replication-namely, hepatitis $\mathrm{B}$ virus DNA and $\mathrm{HbeAg}$-will also appear. IgM anti-HBc, the best serological test for acute hepatitis $\mathrm{B}$, is detectable within 2-4 weeks of the appearance of the surface antigen. If positive, it suggests acute hepatitis $\mathrm{B}$ infection and HbsAg and anti-HBs should be obtained in six months.

The infection may have resolved (HbsAg negative, anti-HBs positive) or may have become chronic (HbsAg positive, anti-HBs negative). If IgM anti-HBc is negative suggesting a chronic hepatitis B virus infection, the HbeAg and anti-HBe will help determine whether the infection in the carrier is actively replicating or is quiescent. ${ }^{6}$ Patients with a positive HBe Ag and hepatitis B virus DNA may be considered for a liver biopsy. Family members and intimate contacts of $\mathrm{HbsAg}$ positive individuals should be screened for exposure to hepatitis B by checking HbsAg and anti-HBc. If anti-HBc negative, family members/intimate contacts would be candidates for immunoprophylaxis if not previously vaccinated. ${ }^{6}$

A positive hepatitis $\mathrm{C}$ antibody in a patient with risk factors would reflect previous contact with the virus. Antibodies to hepatitis $\mathrm{C}$ virus are not protective. The presence of antihepatitis $\mathrm{C}$ in a patient with an abnormal ALT, and risk factors for hepatitis C infection would strongly suggest current hepatitis $\mathrm{C}$ infection. The initial test for antihepatitis $\mathrm{C}$ is the enzyne linked immunosorbent assay (ELISA) test. The test is very sensitive but false positive tests are not infrequent even with third generation ELISAs as seen in hypergammaglobulinaemia of autoimmune hepatitis. If antihepatitis $C$ is negative it is unlikely that the patient has hepatitis C. The specificity of ELISA testing may be improved by addition of the recombinant immunoblot assay for antihepatitis C. ${ }^{3}$ No methods of culturing the virus are available. Consequently detection of the virus in serum is used as a marker of the virus itself. Hepatitis C RNA detected by polymerase chain reaction is the current gold standard. ${ }^{7}$ A positive test would suggest active viral infection and replication. Polymerase chain reaction testing may be done by quantitative or qualitative methods. The sensitivity of quantitative testing varies between 2000200000 viral copies $/ \mathrm{ml}$ whereas qualitative tests will detect as few as 100 viral copies/ml usually within one week of exposure. ${ }^{3}$ Quantitative tests should therefore not be used for diagnosis as these can miss patients with a low level viraemia. Due consideration would be given for antiviral therapy by a specialist. If the polymerase chain reaction is negative despite a positive antibody test, it should be retested in three months to ensure that it was not a false negative.

\section{AUTOIMMUNE HEPATITIS}

Autoimmune hepatitis is an unresolving inflammation of the liver of unknown cause that is associated with interface hepatitis on histological examination, hypergammaglobulinaemia, and autoantibodies. The typical clinical case is that of a young to middle aged woman (a ratio of female to male of 4:1) with raised transaminases in the absence of any other apparent cause. On serum protein electrophoresis around $80 \%$ or more patients demonstrate hypergammaglobulinaemia. ${ }^{8}$ Polyclonal immunoglobulins more than twice normal are highly suggestive. ${ }^{4}$

Appropriate tests would include immunoglobulins, antinuclear antibodies, antibodies to smooth muscle, antiliverkidney microsomal antibodies and antibodies to the soluble liver antigen, which typify type 1,2, and 3 autoimmune liver disease. Routine use of all three tests is not required. Liver biopsy is useful.
Type 1 autoimmune hepatitis is characterised by the presence of antinuclear antibody and/or smooth muscle (actin) antibodies. It is the most common form of the disease worldwide. Seventy percent are women less than 40 years old and over 30\% have concurrent immune diseases, especially autoimmune thyroiditis, synovitis, or ulcerative colitis.

Type 2 autoimmune hepatitis is characterised by the presence of anti-LKMl and is predominantly a disease of children aged 2-14 years. Patients with this form of hepatitis commonly have other concurrent autoimmune disorders such as type 1 diabetes, vitiligo, and autoimmune thyroiditis. Other organ specific autoimmune antibodies such as antibodies to parietal cells, islets of Langerhans, and thyroid may be found. These patients may have low serum levels of immunoglobulin A. Both type 1 and 2 autoimmune hepatitis generally respond well to steroids.

Type 3 autoimmune hepatitis is the least established form of the disease and is characterised by the presence of antibodies to soluble liver antigen/liver pancreas. Patients with these antibodies are indistinguishable from patients with classical type 1 autoimmune hepatitis by age, gender distribution, frequency and nature of other autoantibodies, and responsiveness to steroids.

\section{HEPATIC STEATOSIS AND NON-ALCOHOLIC STEATOHEPATITIS}

Non-alcoholic steatohepatitis (NASH) has emerged in recent years as a chronic hepatic disease of clinical importance. NASH in fact represents a stage within a spectrum of histological disease known as non-alcoholic fatty liver disease. The diagnosis of NASH is usually suspected among individuals with asymptomatic increases in serum liver biochemistries; the presence of a raised body mass index, type 2 diabetes mellitus, or hyperlipidaemia; and no evidence of clinically relevant alcohol use. Mild increases in transaminases (less than four times normal) may be the only clinical clue and in fact it is fair to say that non-alcoholic fatty liver disease is probably the commonest cause of mild aminotransferase increases. ${ }^{9}$ The AST to ALT ratio is usually less than $1: 1 .^{10}{ }^{11}$ Total bilirubin and albumin are usually normal. Leucopenia and thrombocytopenia should raise concerns for the existence of cirrhosis and occult portal hypertension. Ultrasonography, which should form part of the assessment of chronically raised transaminases, may show fatty infiltration. NASH can be proved by liver biopsy ${ }^{4}$ if felt appropriate in the clinical context.

Steatosis tends to follow a benign course whereas NASH may progress to cirrhosis, ${ }^{12}$ though liver failure is uncommon. Programmed weight loss and addressing underlying factors form the basis of treatment. ${ }^{1 .}$

\section{HAEMOCHROMATOSIS}

This is a common autosomal recessive condition among white people associated with increased intestinal absorption of iron and deposition of excessive amounts of iron in the liver, pancreas, and other organs. Many patients are asymptomatic at diagnosis but clinical manifestations include cutaneous hyperpigmentation, diabetes mellitus, and chronic liver disease which may manifest as fatigue, abdominal pain, hepatomegaly, abnormal liver tests, cirrhosis, and hepatocellular carcinoma. Other clinical features include cardiomyopathy, cardiac conduction disorders, hypothyroidism, hypogonadism, impotence, and arthropathy.

A diagnosis of haemochromatosis is based upon a combination of clinical, laboratory, and pathological criteria. A raised serum ferritin raises suspicion of underlying haemochromatosis but a more reliable test is transferrin saturation. Measuring serum iron and total iron binding capacity gives a transferrin saturation index. A value more than $45 \%$ is suggestive. ${ }^{14}$ 


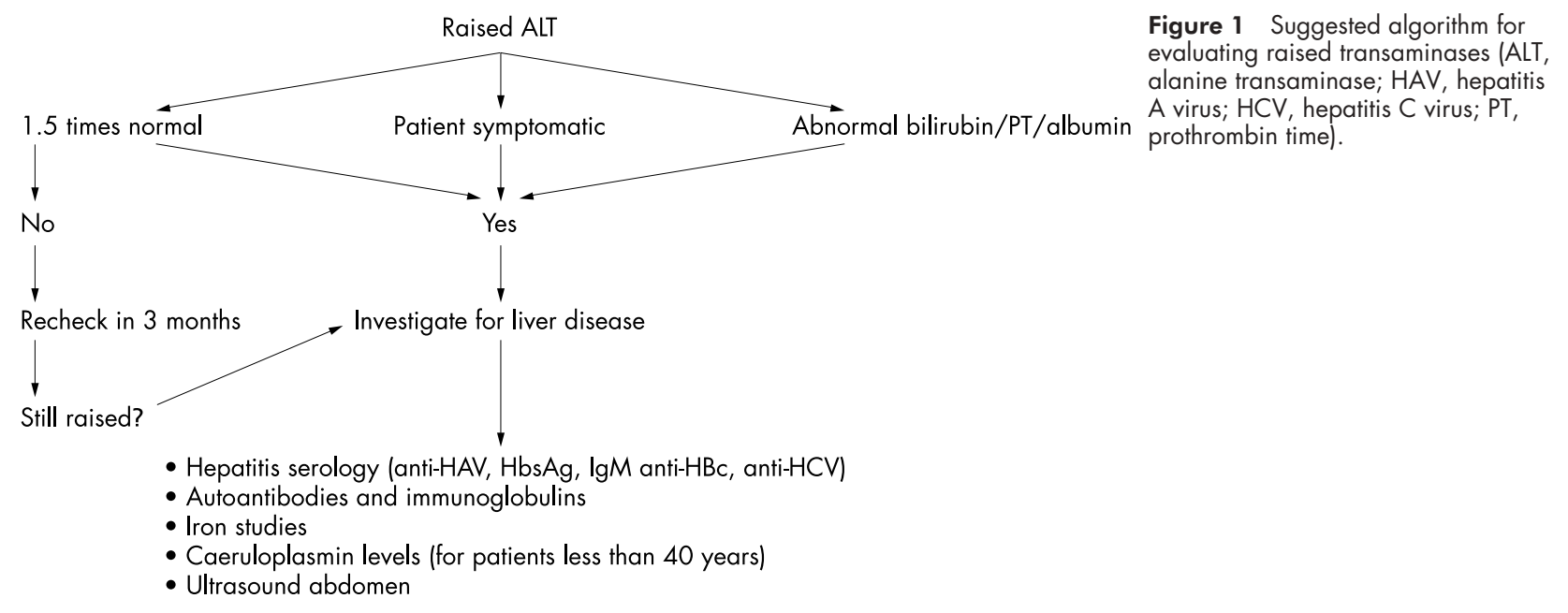

Serum ferritin being an acute phase reactant may also be raised in a number of other inflammatory states and as such is not a useful screening test.

Iron overload may be confirmed by liver biopsy to assess hepatic iron index (hepatic iron level in $\mu \mathrm{mol} / \mathrm{g}$ of dry weight divided by the patient's age). A ratio of more than 1.9 is consistent with homozygous hereditary haemochromatosis. ${ }^{14}$

The discovery of the haemochromatosis gene (HFE) in 1996 has revolutionised the diagnosis of haemochromatosis. The mutation in HFE, located on the short arm of chromosome 6 responsible for the majority of cases is now available by genetic testing. Two point mutations have been designated $\mathrm{C} 282 \mathrm{Y}$ and H63D. The greatest risk of iron overload exists in those who are homozygous for the $\mathrm{C} 282 \mathrm{Y}$ mutation. Iron overload also occurs in a minority of those with other HFE mutations, especially compound heterozygotes who have one copy of C282Y and one copy of H63D and occasionally H63D homozygotes. It must be remembered that clinically significant iron overload can occur in the absence of HFE mutations. A negative gene test therefore does not exclude haemochromatosis. The HFE test is a polymerase chain reaction that is usually performed on a whole blood sample. The gene test is most useful in screening adult family members of an identified proband. It is often also useful in helping resolve ambiguous cases, such as iron overload associated with hepatitis C, alcoholic liver disease, or other causes of end stage liver disease. Before obtaining the HFE test an individual should be counselled about the risks, benefits, and alternatives of genetic testing by a qualified professional. There is concern about the possibility of insurance or employment based on the results of these tests. For this reason gene testing usually is not recommended for anyone younger than 18 years of age.

The need for liver biopsy has decreased with genetic testing becoming available. Liver biopsy still remains the gold standard for assessing the degree of fibrosis. It is important to exclude cirrhosis because of the increased risk of developing hepatocellular carcinoma. In such patients screening with an ultrasound and $\alpha$-fetoprotein every six months may be appropriate. A recent study ${ }^{15}$ confirmed that certain non-invasive predictors were accurate in excluding cirrhosis in $\mathrm{C} 282 \mathrm{Y}$ homozygotes. In this study, there were no cases of cirrhosis in 96 C282Y homozygotes who had serum ferritin levels lower than $1000 \mu \mathrm{g} / \mathrm{l}$, normal AST values, and no evidence of hepatomegaly. These results have been confirmed in other studies. A serum ferritin of $<1000 \mu \mathrm{g} / \mathrm{l}$ therefore seems to be the best predictor of the absence of cirrhosis in $\mathrm{C} 282 \mathrm{Y}$ homozygotes. The positive predictive value of a serum ferritin $>1000 \mu \mathrm{g} / \mathrm{l}$ is poor, however, as only about $50 \%$ of those with values of $>1000 \mu \mathrm{g} / \mathrm{l}$ have cirrhosis. A liver biopsy is advisable in this group of patients to definitely assess for the presence of cirrhosis. Similar information is not available for non-C282Y homozygotes.
Liver biopsy is not essential in patients with hereditary haemochromatosis less than 40 years with normal liver function. ${ }^{4}$

\section{WILSON'S DISEASE}

This is a genetic disorder of biliary copper excretion. Usually detected between the ages of 5 and 25, it should also be considered in patients up to 40 years of age.

Serum caeruloplasmin, the usual screening test is reduced in approximately $85 \%$ of cases. ${ }^{4}$ Kayser-Fleischer rings may be a useful clinical clue. Caeruloplasmin may be normal and Kayser-Fleischer rings absent, in which case a 24 hour urinary copper excretion should be checked. Excretion of more than $100 \mu \mathrm{g}$ suggests Wilson's disease.

Liver biopsy will confirm the diagnosis if hepatic copper concentrations are more than $250 \mu \mathrm{g} / \mathrm{g}$ dry liver weight. The genetic defect has been identified but due to the large number of mutations involved molecular diagnosis is not yet possible.

\section{ALPHA $_{1}$-ANTITRYPSIN DEFICIENCY}

This is an uncommon cause of chronic liver disease in adults. Low levels of $\alpha_{1}$-antitrypsin may be detected by direct measurement of serum levels or by the lack of a rise in $\alpha$-globulin bands on serum protein electrophoresis. ${ }^{4}$

\section{NON-HEPATIC CAUSES OF ABNORMALITY}

Hepatic infiltration such as metastatic or even primary hepatocellular carcinoma, tuberculosis, sarcoidosis, and amyloidosis may cause a modest (up to threefold) rises in aminotransferases, and an up to 20-fold rise in ALP depending upon the extent of involvement. Bilirubin levels are usually normal but up to fivefold increases are recognised. ${ }^{3}$

Occult coeliac sprue is recognised as a cause of raised transaminases. ${ }^{16}$ Antiendomyseal antibodies and antigliadin antibodies are useful confirmatory tests. Box 4 lists common and rare but recognised non-hepatic causes of transaminase abnormalities.

If despite comprehensive testing the cause is not apparent a liver biopsy may be required. Figure 1 suggests an algorithm for the investigation of abnormal transaminases.

\section{CAUSES OF RAISED ALKALINE PHOSPHATASE}

ALP originates mainly from two sources: liver and bone. ${ }^{2}$ The enzymes may be present in a variety of other tissues namely intestine, kidney placenta, and leucocytes. The elevation may be physiological or pathological. The physiological role of these enzymes is not entirely clear but production increases in tissues undergoing metabolic stimulation. Elevations are seen 


\section{Box 5: Common causes of raised ALP}

(A) Physiological

- Women in the third trimester of pregnancy.

- Adolescents.

- Benign, familial (due to increased intestinal ALP).

(B) Pathological

- Bile duct obstruction.

- Primary biliary cirrhosis.

- Primary sclerosing cholangitis.

- Drug induced cholestasis-for example, anabolic steroids.

- Adult bile ductopenia.

- Metastatic liver disease.

- Bone disease.

in the third trimester of pregnancy, a result of an influx of placental ALP. Adolescents may show increases that are twice normal for adults as a result of bone ALP into the blood, corresponding with growth.

Clearly the first step in determining the cause is to identify the source of the raised ALP. The most sensitive and indeed specific method is electrophoretic separation but it is not freely available. A good discriminator is testing for 5' nucleotidase or gammaglutamyl transferase, which rise in liver but not bone disease. If ALP is of liver origin, imaging by ultrasound should be arranged.

Box 5 lists common causes of raised ALP. Extrahepatic biliary obstruction, infiltrative diseases, and metastasis may cause mild to striking elevations in ALP

Hepatic ALP is present on the canalicular and luminal domain of the bile duct epithelium and levels rise as a result of increased synthesis and consequent release into the circulation. As a result levels may not rise until one or two days after biliary obstruction. Further the enzyme has a half life of a week and so even after resolution of biliary obstruction, it may take several days for levels to normalise.

It is also worth noting that ALP may be raised in malignancies without liver or bone involvement. This isoenzyme is called the "Regan isoenzyme" and occurs in various neoplasms for example lung cancers. ${ }^{3}$
Box 6: Causes of raised gammaglutamyl transferase

- Hepatobiliary disease (often with other liver enzyme abnormalities).

- Pancreatic disease.

- Alcoholism.

- Chronic obstructive pulmonary disease.

- Renal failure.

- Diabetes.

- Myocardial infarction.

- Drugs-for example, carbamazepine, phenytoin, and barbiturates.

Figure 2 suggests an algorithm for evaluating patients with a raised ALP.

\section{GAMMAGLUTAMYL TRANSFERASE}

This enzyme is found in hepatocytes and biliary epithelial cells. Though a sensitive test of hepatobiliary disease its usefulness is limited by lack of specificity. Raised levels may be seen in pancreatic disease, myocardial infarction, renal failure, chronic obstructive pulmonary disease, diabetes, and alcoholism. ${ }^{17}$

Medications like phenytoin, carbamazepine, and barbiturates may also cause a mild rise in gammaglutamyl transferase (box 6). ${ }^{18}$

With other enzyme abnormalities, a raised gammaglutamyl transferase would support a hepatobiliary source. For example it would confirm hepatic source for a raised ALP. A raised gammaglutamyl transferase with raised transaminases and a ratio of AST to ALT of 2:1 or more would suggest alcohol related liver disease.

A sensible approach is to follow patients with isolated gammaglutamyl transferase elevations at few monthly intervals. If other enzymes become abnormal or gammaglutamyl transferase increases further an abdominal ultrasound, computed tomography, or both should be requested to exclude a space occupying lesion and a liver biopsy examination considered. ${ }^{19}$

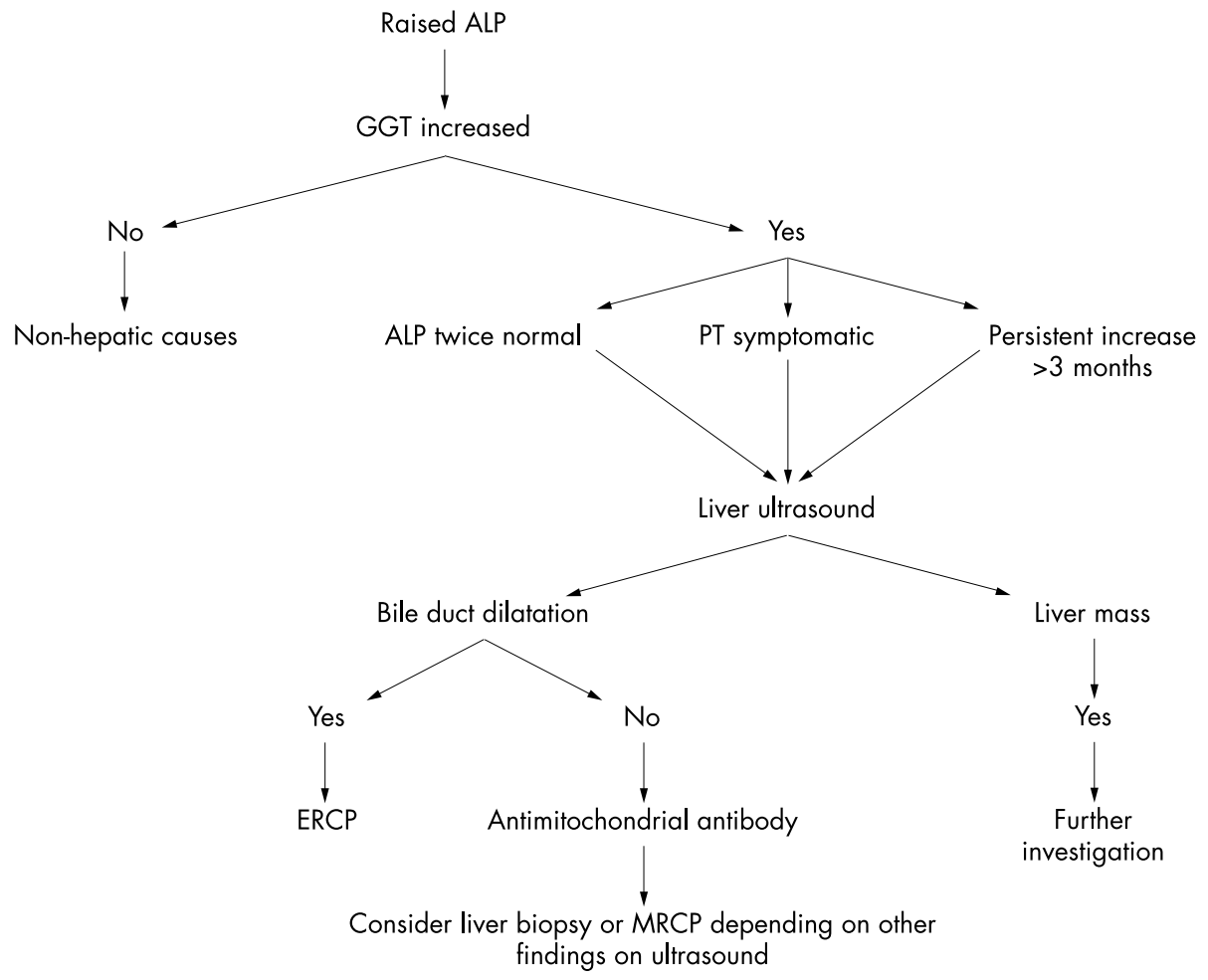

Figure 2 Suggested algorithm for evaluating a raised ALP (ALP, alkaline phosphatase; ERCP, endoscopic retrograde

cholangiopancreatography; GGT, gammaglutamyl transferase; PT, prothrombin time; $M R C P$, magnetic resonance cholangiopancreatography). 


\section{ALBUMIN}

Albumin synthesis is an important function of the liver Approximately $10 \mathrm{~g}$ is synthesised and secreted daily. With progressive liver disease serum albumin levels fall, reflecting decreased synthesis. Albumin levels are dependant on a number of other factors such as the nutritional status, catabolism, hormonal factors, and urinary and gastrointestinal losses. These should be taken into account when interpreting low albumin levels. Having said that, albumin concentration does correlate with the prognosis in chronic liver disease.

\section{PROTHROMBIN TIME}

The synthesis of coagulation factors (except factor VIII) is an important function of the liver. The prothrombin time measures the rate of conversion of prothrombin to thrombin (requiring factors II, V, VII, and X) and thus reflects a vital synthetic function of the liver. Vitamin $\mathrm{K}$ is required for the gamma carboxylation of the above named factors.

Prothrombin time may therefore be prolonged in vitamin $\mathrm{K}$ deficiency, warfarin therapy, liver disease, and consumptive coagulopathy.

It is important to distinguish a prolonged prothrombin time due to hepatocellular disease from that due to chronic cholestasis with fat malabsorption. In practice a useful way of differentiating the two is the administration of vitamin $\mathrm{K}$, which will reduce a prolonged prothrombin time due to fat malabsorption but not due to intrinsic liver disease.

\section{INTERNATIONAL NORMALISED RATIO}

This is more often tested now instead of or along with prothrombin time, in order to standardise the reporting of prothrombin time (PT) results. It is calculated according to a formula as follows:

International normalised ratio $=[$ patient PT $/$ mean control PT] ${ }^{\text {ISI }}$

(ISI $=$ international sensitivity index).

This is helpful because it avoids the interlaboratory variability in prothrombin time. Its interpretation is otherwise similar to prothrombin time.

\section{QUANTITATIVE TESTS OF LIVER FUNCTION}

Limitations of the various biochemical tests have prompted the search for more sensitive and quantitative tests of liver function. Though these tests are currently limited to research centres they deserve mention and include ${ }^{3}$ :

- Indocyanine green clearance.

- ${ }^{14} \mathrm{C}$-aminopyrine breath test.

- Antipyrine clearance.

- Galactose elimination capacity.

- ${ }^{13} \mathrm{C}$-caffeine breath test.

The tests are expensive and more labour intensive. Well designed clinical trials are needed comparing them to biochemical tests before they gain wider acceptance.

\section{WHEN TO REFER FOR A SPECIALIST OPINION?}

This would normally include ${ }^{6}$ patients with:

(1) Unexplained liver abnormalities more than 1.5 times normal on two occasions, a minimum of six months apart.

(2) Unexplained liver disease with evidence of hepatic dysfunction (hypoalbuminaemia, hyperbilirubinaemia, prolonged prothrombin time, or international normalised ratio)

(3) Known liver disease where treatment beyond the withdrawal of the implicating agent is required.

\section{WHAT TESTS TO DO BEFORE REFERRAL ${ }^{6}$}

Consider the following:

(1) Screen for viral hepatitis: IgM antihepatitis A virus, $\mathrm{HbsAg}$, antihepatitis C virus.

\section{Key points}

- Abnormal liver tests may present in an asymptomatic patient.

- A good clinical history and physical examination are often rewarding.

- Liver tests often become abnormal in non-hepatic diseases.

- If a systematic approach is adopted the cause is often apparent.

- A specialist opinion should be sought when appropriate.

(2) Antinuclear antibodies

(3) Caeruloplasmin in patients younger than 40 years.

(4) Iron studies (iron, total iron binding capacity, and ferritin).

(5) Ultrasound of the liver especially where fatty infiltration is suspected (obese individuals, diabetics and/or hyperlipidaemic patients).

An ultrasound should also be performed in symptomatic patients with liver enzyme abnormalities or those with evidence of hepatic dysfunction (increased bilirubin or prothrombin time, or decreased albumin) and in those with biochemical evidence of cholestasis.

\section{Authors' affiliations}

J K Limdi, Tameside General Hospital, Ashton-under-Lyne, Manchester G M Hyde, Wythenshawe Hospital, South Manchester University Hospitals NHS Trust, Wythenshawe, Manchester

\section{REFERENCES}

1 Friedman LS, Martin P, Munroz SJ. Liver function tests and the objective evaluation of the patient with liver disease. In: Zakim D, Boyer TD, ed. Hepatology: a textbook of liver disease. Vol 1. Philadelphia, WB Saunders, 1996: 791-833.

2 Pratt DS, Kaplan MM. Laboratory tests. In: Schiff ER, Sorrell MF, Maddrey WC, eds. Schiff's diseases of the liver. 8th Ed. Vol 1. Philadelphia: Lippencott-Raven, 1999: 205-44.

3 Feldman M, Friedman LS, Sleisenger MH. Sleisenger \& Fordtran's gastrointestinal and liver disease. 7th Ed. 2002: 1227-39, 1310-11.

4 Pratt DS, Kaplan MM. Evaluation of abnormal liver enzyme results in asymptomatic patients. N Engl J Med 2000;342:1266-71.

5 Cohen JA, Kaplan MM. The SGOT/SGPT ratio-an indicator of alcoholic liver disease. Dig Dis Sci 1979;24:835-8.

6 Minuk G. Canadian Association of Gastroenterology Practice Guidelines: evaluation of abnormal liver enzyme tests. Can J Gastroenterol 1998;12(6):417-21.

7 Schiff ER, de Medina M, Kahn RS. New perspectives in the diagnosis of hepatitis C. Semin Liver Dis 1999; suppl 1:3-15

8 Czaja AJ. Natural history, clinical features, and treatment of autoimmune hepatitis. Semin Liver Dis 1984;4:1-12.

9 Diehl AM Goodman Z, Ishak KG. Alcohol like liver disease in nonalcoholics: a clinical and histologic comparison with alcohol-induced liver injury. Gastroenterology 1988;95:1056-62.

10 Bacon BR, Farahvash M, Janney CG, et al. Non-alcoholic steatohepatitis: an expanded clinical entity. Gastroenterology 1994;107:1 103-9.

11 Sorbi D, Boynton J, Lindor KD. The ratio of aspartate aminotransferase to alanine transferase: potential value in differentiating non-alcoholic steatohepatitis from alcoholic liver disease. Am J Gastroenterol 1999:94:1018-22

12 Matteoni CA, Younossi ZM, Gramlich T, et al. Nonalcoholic fatty liver disease: a spectrum of clinical and pathological severity. Gastroenterology 1999;116:1413-9.

13 Eriksson S, Eriksson KF, Bondesson L.Nonalcoholic steatohepatitis in obesity: a reversible condition. Acta Med Scand 1986:220:83-8.

14 Powell LW, George DK, McDonnell SM, et al. Diagnosis of haemochromatosis. Ann Intern Med 1998;129:925-31.

15 Guyader D, Jacquelinet C, Moirand R, et al. Non invasive prediction of fibrosis in $\mathrm{C} 282 \mathrm{Y}$ homozygous haemochromatosis. Gastroenterology 1998; 115:929-36

16 Bardella MT, Vecchi M, Conte D, et al. Chronic unexplained hypertransaminasemia may be caused by occult coeliac disease. hypertransaminasemia may be
Hepatology 1999;29:654-7.

17 Goldberg DM, Martin JV. Role of gamma-glutamyl transpeptidase activity in the diagnosis of hepatobiliary disease. Digestion $1975 ; 12: 232-46$

18 Rosalki SB, Tarlow D, Rau D. Plasma gamma-glutamyl transpeptidase elevation in patients receiving enzyme-inducing drugs. Lancet 1971;ii:376-7.

19 Bloom S, ed. Abnormal liver function test in an asymptomatic patient. Practical gastroenterology. 1st Ed. UK: Martin Dunitz, 2002: 503-6. 